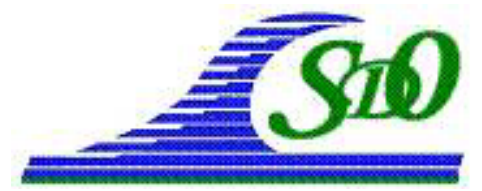

XI ìmes Journées Nationales Génie Côtier - Génie Civil

Les Sables d'Olonne, 22-25 juin 2010

DOI:10.5150/jngcgc.2010.049-S C Editions Paralia CFL

disponible en ligne - http://www.paralia.fr - available online

\title{
Modélisation numérique de la re-suspension de sédiments induite par la navigation
}

\author{
Hassan SMAOUI ${ }^{1}$, Abdellatif OUAHSINE ${ }^{2}$, \\ Damien PHAM VAN BANG ${ }^{3}$, Philippe SERGENT ${ }^{4}$, \\ François HISSEL ${ }^{4}$
}

1. CETMEF-LHN, Laboratoire Roberval, UMR-CNRS 6253, B.P. 20529, 60206 Compiègne Cedex, France.

Hassan.Smaoui@developpement-durable.gouv.fr

2. UTC-LHN, Laboratoire Roberval, UMR-CNRS 6253, B.P. 20529, 60206 Compiègne Cedex, France.

3. CETMEF-EDF/R\&D, Laboratoire d'Hydraulique Saint Venant

6, quai Watier, Bat O, B.P.49, 78401 Chatou Cedex, France.

4. Centre d'Etudes Techniques Maritimes Et Fluviales (CETMEF),

2 Bd Gambetta, B.P.60039, 60321 Compiègne Cedex, France.

\section{Résumé :}

Cette étude s'intéresse à la remise en suspension de sédiments occasionnée par le passage de bateaux. En complément à des mesures in situ, une modélisation 1DV (1D Vertical) est proposée pour estimer les paramètres qui se sont révélés mal déterminés. Le modèle résout la structure verticale de la vitesse horizontale $(u)$, de la concentration en MES (matières en suspension) (c), de l'Energie Cinétique Turbulente $(k)$ et sa longueur de mélange $(L)$. La turbulence est modélisée par un modèle à deux équations de type $(k-k L)$, et le forçage est effectué à la surface libre par les passages de bateaux. Les résultats montrent que l'hypothèse d'un écoulement cisaillé à la surface est satisfaisante pour simuler les pics de MES générés par les bateaux.

Mots-clés :

Transport fluvial - Transport sédimentaire - Turbulence - Modélisation

\section{Introduction}

Au début du vingtième siècle, le réseau français des voies navigables est arrivé à son apogée puisqu'il comptait $12800 \mathrm{~km}$ entre fleuves et canaux. Puis la concurrence de la voie ferrée et du réseau routier ont causé son déclin.

Si le trafic fluvial a augmenté de 30\% en dix ans (1997-2007), il ne représente toujours que 3\% des modes de transport. Et le livre blanc de la commission européenne de 2001 le qualifiait de "sous-exploité" bien qu'il soit un "élément clé" de l'inter modalité. Plus récemment, le Grenelle de l'environnement a relancé le débat, en préconisant un report ambitieux de $25 \%$ de la route vers le fleuve à l'horizon 2020 . 
En effet, le transport fluvial répond pleinement aux défis du vingt et unième siècle en termes de développement durable. Au-delà des réductions d'émissions de gaz à effet de serre, il présente aussi des avantages significatifs tels que l'absence de nuisances sonores ou visuelles, une meilleure sécurité et la dé-saturation des axes routiers. Mais le report modal vers le fluvial aura certainement des conséquences économiques et écologiques. Le changement de gabarits et de la fréquence modifiera l'hydrodynamique (courants de retours et ondes de batillage) et la dynamique sédimentaire (érosion des fonds et des berges). Or ces derniers conditionnent la qualité du milieu aquatique ainsi que l'intégrité des propriétés riveraines. A ces impacts économiques et environnementaux s'ajoutent enfin des coûts pour l'entretien et la restauration des voies de navigation.

Donc, l'impact du passage des bateaux sur le transport sédimentaire et l'érosion des berges a été mesuré sur deux sites en 2006 (PHAM VAN BANG et al., 2007 ; PHAM VAN BANG et al., 2008). Des courantomètres, des turbidimètres et des jauges de hauteur d'eau ont été déployés. Les résultats expérimentaux servent ici à proposer un modèle numérique simple permettant de simuler la re-suspension des sédiments aux passages de bateaux.

Puisque les mesures effectuées ne suffisent pas pour quantifier toutes les variables du problème, l'application du modèle $1 \mathrm{DV}$ est pertinent pour les évaluer (PARCHURE et al., 2001). Pour des hypothèses simplificatrices, la distribution verticale des fluctuations turbulentes sous les bateaux est ainsi prédite. Dans cet article, nous présentons l'approche numérique en commençant par la description du modèle mathématique 1DV et son application aux sites de la campagne de mesures. Puis, nous analysons à partir des résultats du modèle, l'impact du passage des bateaux (en régime sans hélice) sur la remise en suspension avec ou sans les effets cumulés des flux sédimentaires.

\section{Présentation du modèle mathématique}

Le modèle 1DV utilisé dans cette étude est basé sur la version 3D des modèles d'écoulements à surface libre mais dans lequel tous les gradients horizontaux ont été négligés, excepté le gradient de pression. Il est constitué d'une équation de la quantité de mouvement, deux équations pour les variables turbulentes et une équation de transport de la matière en suspension. Elles sont données par :

$$
\begin{aligned}
& \frac{\partial u}{\partial t}+\frac{1}{\rho} \frac{\partial p}{\partial x}=\frac{\partial}{\partial z}\left[\left(v+v_{t}\right) \frac{\partial u}{\partial z}\right] \\
& \frac{\partial k}{\partial t}=v_{t}\left(\frac{\partial u}{\partial z}\right)^{2}+\frac{\partial}{\partial z}\left[v_{t k} \frac{\partial k}{\partial z}\right]-\frac{k \sqrt{k}}{B_{1} L} \\
& \frac{\partial k L}{\partial t}=A_{l} L v_{t}\left(\frac{\partial u}{\partial z}\right)^{2}+\frac{\partial}{\partial z}\left[v_{t k} \frac{\partial k L}{\partial z}\right]-\frac{k L^{2} \sqrt{k}}{B_{2} \tilde{w}} \\
& \frac{\partial c}{\partial t}=w_{c} \frac{\partial c}{\partial z}+\frac{\partial}{\partial z}\left[\gamma_{t} \frac{\partial c}{\partial z}\right]
\end{aligned}
$$


où $t$ est le temps, $x$ la coordonnée horizontale, $z$ la coordonnée verticale, $u$ la vitesse horizontale, $p$ la pression, $\rho$ la densité de l'eau, $v$ la viscosité moléculaire, $v_{t}$ la viscosité turbulente, $k$ l'énergie cinétique turbulente (ECT), $L$ la longueur de mélange, $v_{t k}$ la diffusivité turbulente de l'ECT, $\widetilde{w}$ la fonction de paroi. $\left(A_{1}, B_{1}, B_{2}\right)$ sont des constantes empiriques (tableau 1), et $w_{c}$ la vitesse de chute des sédiments de diamètres inférieurs à $63 \mu \mathrm{m}$ est donnée par :

$w_{c}=\frac{g d^{2}(s-1)}{18 v}$

où $g$ est l'accélération de la gravité, $d$ le diamètre des sédiments, $s=\rho_{s} / \rho$ le rapport de densité des sédiments.

Pour fermer le système, BLUMBERG et MELLOR (1987), ont donné la viscosité et la diffusivité turbulente en fonction de l'ECT $k$, de la longueur de mélange $L$ et des fonctions de stabilité $S_{m}, S_{k}$ et $S_{h}$ ) (tableau 1):

$v_{t}=L \sqrt{k} S_{m} \quad, \quad v_{t k}=L \sqrt{k} S_{k} \quad, \quad \gamma_{t}=\frac{S_{h}}{S_{m}} v_{t}$

La résolution numérique du système d'équations (1-4) avec (5-6) nécessite la donnée des conditions initiales et aux limites (au fond $z=z_{0}$ et à la surface $z=H$ ).

Tableau 1. Constantes empiriques du modèle de la turbulence $k$ - $k L$

\begin{tabular}{llllll}
\hline$A_{1}$ & $B_{1}$ & $B_{2}$ & $S_{h}$ & $S_{k}$ & $S_{m}$ \\
\hline 1.80 & 16,6 & 12,48 & 0.49 & 0.20 & 0.39 \\
\hline
\end{tabular}

\subsection{Conditions au fond}

Pour la vitesse $u$, on suppose la condition de non glissement $\left(u\left(z_{0}\right)=0\right)$. L'équilibre entre la production et la dissipation de l'ECT et une loi linéaire pour la longueur de mélange sont également admis. Sous ces hypothèses, les conditions à imposer sont alors $k\left(z_{0}\right)=\alpha_{l} u_{*}^{2}$ avec $\alpha_{l}=6,74$ et $L\left(z_{0}\right)=\kappa z_{0}$, où $u_{*}$ est la vitesse de frottement et $\kappa$ est la constante de Karman.

La condition au fond pour la MES porte sur le flux total à travers l'interface fluide-fond. Ce flux s'écrit comme la différence entre le flux descendant $D$ (ou de dépôt) dû à la sédimentation et le flux ascendant $E$ (ou d'érosion) dû à un excès de contrainte de cisaillement. Cette condition s'écrit alors par :

$\left(\gamma_{\mathrm{t}} \frac{\partial \mathrm{c}}{\partial \mathrm{z}}+\mathrm{w}_{\mathrm{c}} \mathrm{c}\right)_{\mathrm{z}=\mathrm{z}_{0}}=\mathrm{D}-\mathrm{E}$

Le taux du dépôt $D$ est considéré proportionnel à la concentration de la MES près du fond avec la vitesse de chute $w_{c}$ comme coefficient de proportionnalité :

$D=w_{c} c\left(z_{0}\right)$

Par ailleurs, le taux d'érosion $E$, en situation d'équilibre et sous certaines hypothèses selon CELIK et RODI $(1984,1988,1991)$ et VAN RIJN (1986), peut être calculé par : 
$E=w_{c} c_{b} \frac{\gamma_{0} S}{1+\gamma_{0} S}$

où $c_{b}$ est la concentration volumique du sédiment ( $c_{b}=0.65$ pour du sable), $\gamma_{0}$ est une constante empirique de la re-suspension et $S$ est l'excès de contrainte de cisaillement normalisé $\left(\tau_{b}\right)$ par rapport à une valeur critique $\left(\tau_{c r i}\right)$ :

$S=\frac{\tau_{b}-\tau_{c r i}}{\tau_{c r i}}$

\subsection{Conditions à la surface}

A la surface libre, nous considérons, en première approximation et en négligeant les effets d'hélices, que les vitesses des bateaux sont transmises par cisaillement directement à la couche de surface $\left(u(H)=V_{\text {bateau }}\right)$. L'équilibre entre production et dissipation de l'ECT, et une loi linéaire pour la longueur de mélange sont également admis à $\mathrm{z}=\mathrm{H}$. Ainsi, les conditions à imposer à la surface libre sont : $k(H)=\alpha_{l} u_{*_{s}}^{2}$ et $L(H)=\kappa z_{s}$, où $u_{*, s}$ est la vitesse de frottement et $z_{s}$ est la rugosité de la surface.

La condition limite pour la MES exprime la nullité du flux à travers la surface :

$\left(\gamma_{t} \frac{\partial c}{\partial z}+w_{c} c\right)_{z=H}=0$

\subsection{Résolution numérique}

L'écoulement que nous modélisons présente une couche limite à la surface et au fond. Nous avons donc utilisé un maillage en trois couches (de fond, intermédiaire et de surface) avec un raffinement (de l'ordre du centimètre) pour les couches de fond et de surface. Afin de limiter les procédures d'interpolation, les variables moyennes $(u, c)$ et les variables turbulentes $\left(k, L, v_{t}, \gamma_{t}\right)$ sont calculées sur deux grilles décalées. Ceci permet d'exprimer de manière naturelle les différents gradients.

La résolution du système d'équations (1-4) avec leurs conditions aux limites a été discrétisé par la méthode des volumes finis en espace (schéma centré, sauf pour le terme convectif) et par des différences finies en temps (schéma d'Euler). Notons que la discrétisation des équations a abouti à la résolution d'un système linéaire de type tridiagonal par l'algorithme de Thomas (TDMA : TriDiagonal Matrix Algorithm).

\section{Présentation succincte des données expérimentales}

Le passage d'une centaine de bateaux a été enregistré sur deux sites (le premier sur le canal de la Sensée et le second sur la Seine) par des observations visuelles (relevé du trafic) et des mesures subaquatiques. Ces dernières sont obtenues à partir de cinq OBS (Optical Backscatter Sensor) dont un est ancré à $10 \mathrm{~cm}$ du fond pour mesurer la MES au centre du chenal, deux capteurs $\mathrm{S} 4$ (courantomètre et baromètre) positionnés à $15 \mathrm{~cm}$ du 
fond. Les détails de l'expérimentation, des résultats et des analyses sont reportés dans PHAM VAN BANG et al., (2007, 2008).

Parallèlement à ces mesures subaquatiques, le trafic fluvial (sens, vitesses, chargements, trajectoires) a été relevé. Ces dernières informations servent à constituer une chronique pour forcer la variable vitesse de l'équation (1) du modèle 1DV.

Enfin, des prélèvements sur le fond ont été réalisés pour les deux sites. Des analyses réalisées par le Laboratoire Régional de l'Est Parisien (LREP), il ressort que les deux sites sont constitués essentiellement de sable-vase de diamètre $d_{50}$ entre $20 \mu \mathrm{m}$ et 40 $\mu \mathrm{m}$. Cette valeur est utilisée pour estimer la rugosité du fond $z_{0} \cong 8 d_{50}$.

\section{Présentation des résultats de simulations}

Une centaine de passage de bateaux a été enregistrée lors de la campagne de mesures. Nous présentons ici, à titre d'exemples, les simulations des journées du 08-09 novembre 2006 (site 1) et du 15 novembre 2006 (site 2).

\subsection{Les vitesses induites par les bateaux}

La figure 1 présente les vitesses et les variables liées à la turbulence simulées par le modèle sur deux cas de bateaux (D1 avalant et D9 montant) de la journée du 08/11 de vitesses respectivement $13,5 \mathrm{~km} / \mathrm{h}$ et $8,0 \mathrm{~km} / \mathrm{h}$. Ces vitesses ont été enregistrées respectivement à $9 \mathrm{~h} 45 \mathrm{mn}$ et $12 \mathrm{~h} 20 \mathrm{mn}$ GMT. Les résultats du modèle reproduisent correctement les profils de vitesse notamment le courant de retour près du fond (renversement du profil). Les figures 1.b, 1.c et 1.d illustrent les profils verticaux des variables $\left(k, L, v_{t}\right)$ en accord avec la théorie.

Grâce à sa bonne résolution à proximité du fond (section 2.3), le modèle 1DV fournit des mesures de vitesse à $10 \mathrm{~cm}$ et à $25 \mathrm{~cm}$ du fond pour tout le trafic journalier du 08/11 (site 1). La chronique obtenue présente des pics fortement irréguliers. Ils sont attribués aux différentes conditions de navigation. Pour cette journée, la moyenne des pics de vitesse s'élève à $3,5 \mathrm{~cm} / \mathrm{s}$ à $z=10 \mathrm{~cm}$ et à $4,4 \mathrm{~cm} / \mathrm{s}$ à $z=25 \mathrm{~cm}$ depuis le fond. Ces ordres de grandeur sont en accord parfait avec les mesures in situ. Pour la journée du 15/11 (site 2), le modèle fournit une vitesse de $12 \mathrm{~cm} / \mathrm{s}$ à $z=10 \mathrm{~cm}$ et de $16 \mathrm{~cm} / \mathrm{s}$ à $z=25 \mathrm{~cm}$. Cet écart est sans doute expliqué par l'effet de site et/ou du type de bateaux.

A partir de ces résultats (figure 1), le modèle numérique estime la contrainte de cisaillement au fond $\tau_{b}$, cette dernière n'ayant pas pu être quantifiée précisément faute d'une instrumentation dédiée. Le modèle estime $\tau_{b}$ à $0,43 \mathrm{~N} \mathrm{~m}^{-2}$ pour la journée du $08 / 11$ (site 1) et à $1,14 \mathrm{~N} \mathrm{~m}^{-2}$ pour celle du $15 / 11$ (site 2). Cette dernière valeur est du même ordre de grandeur que les valeurs publiées par VERNEY et al. (2007) dans la partie estuarienne de la Seine ( $\tau_{b}$ est entre 1 et $2 \mathrm{~N} \mathrm{~m}^{-2}$ ). 

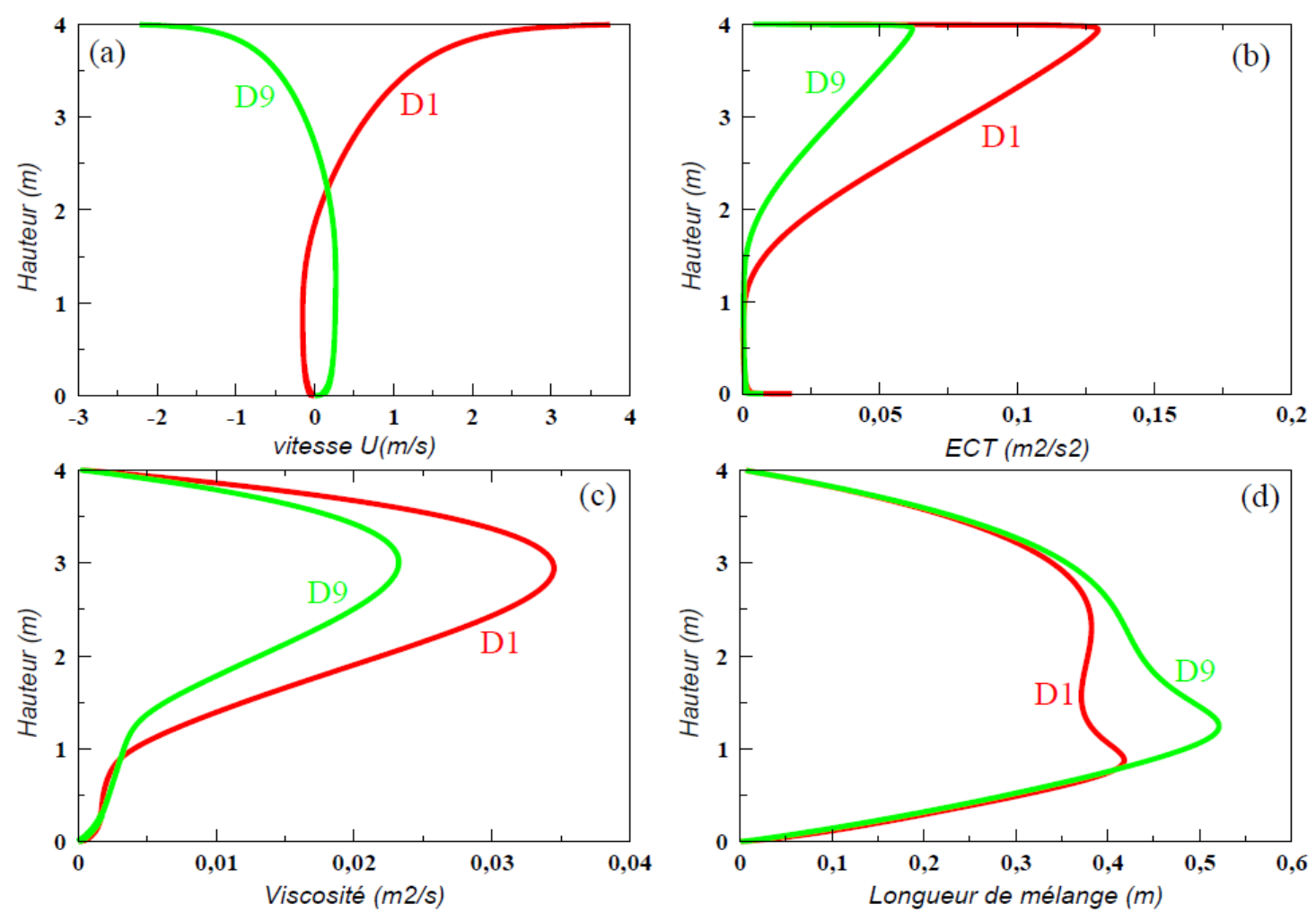

Figure 1. Profils verticaux issus de la simulation. (a) Vitesse; (b) Energie cinétique turbulente; (c) Viscosité turbulente ; (d) Longueur de mélange.

\subsection{Re-suspension des sédiments induite par les bateaux}

La figure 2.a présente la superposition des chroniques de la concentration en MES (à 10 $\mathrm{cm}$ du fond) simulées et mesurées sur le site 1. L'asymétrie des pics de re-suspension observée in situ est fidèlement reproduite. Elle met en évidence un régime rapide d'érosion suivi d'un régime lent de sédimentation.

Les amplitudes et les durées des pics de concentration (figure 2) obtenus par le modèle 1DV sont en étroite concordance avec les enregistrements sur le terrain. A titre d'exemple pour la journée du 10/11/2006, le maximum de la valeur absolue de la différence entre les pics simulés et mesurés ne dépasse pas $3 \times 10^{-3} \mathrm{~g} / 1$.

La figure 2, montre que le modèle sous estime par rapport aux mesures la concentration de la MES lors de la décroissance (ou de la sédimentation). En effet, le modèle ne calcule que la concentration des sédiments de diamètre moyen de $40 \mu \mathrm{m}$, alors que les OBS mesurent la totalité des classes en suspension constituant la couverture sédimentaire du lit. Par conséquent les classes inférieures à $40 \mu \mathrm{m}$ que le modèle ignore restent plus longtemps en suspension. A cela s'ajoute les effets des hélices (que le modèle ne prend pas en compte) qui peuvent être source de la turbulence favorisant ainsi la mise en suspension. 


\section{XI $I^{\text {mes }}$ Journées Nationales Génie Côtier - Génie Civil}

Les Sables d'Olonne, 22-25 juin 2010

Notons que le modèle 1DV permet de renseigner sur les amplitudes et la durée des pics de la MES sans passer par aucune hypothèse simplificatrice. En effet, sur les figures 2 de la concentration de la MES, on peut directement lire sur les courbes, les temps caractéristiques de la décroissance noté $\tau$. A titre d'exemple, on peut lire $\tau_{\mathrm{D} 59}$ entre 10 et $12 \mathrm{mn}$ pour le bateau D59 de la journée du 10/11/2006 (figure 2d). Ces ordres de grandeur sont en correspondance avec les résultats issus des mesures (figure $2 \mathrm{~d}$ ).

Sur la figure 3, nous présentons les profils de la concentration de la MES induits par le passage des bateaux D1 et D9 du trafic pour le site 1. En première approximation, ces profils sont conformes à la loi de Rouse (solution analytique en loi exponentielle en $z$ ).

Nous observons également que la remise en suspension concerne essentiellement une couche de fond d'une cinquantaine de centimètres. Précisons que dans cette couche ce sont principalement les vitesses de retour des bateaux qui agissent. Ainsi, pour une bonne estimation de la concentration en MES, les modèles numériques devront calculer avec précision ces vitesses de retour.

\section{Conclusions}

La prédiction du mouvement des sédiments est un exercice très difficile, mais essentiel pour les études d'ordre écologique et d'ordre économique. Ces études ont été réalisées par mesure in situ (canal de la Sensée et la Seine) pour caractériser l'impact de la navigation sur le transport sédimentaire. Dans la continuité de ces travaux, nous avons développé un modèle numérique 1DV simple, mais complet (hydrodynamique, turbulence, transport sédimentaire) pour simuler les mouvements des sédiments lors des passages des bateaux. Basé sur des hypothèses simplificatrices, le modèle reproduit correctement les caractéristiques d'un écoulement au-dessous d'un bateau en régime sans hélice. Il est alors capable de prédire raisonnablement les effets des passages de bateaux sur le fond.

Enfin, notons que le modèle présenté ici ne rend pas compte ni de l'écoulement global au-dessous d'un bateau, ni de transport sédimentaire vaseux. Un modèle numérique (hydrodynamique, turbulence, transport sédimentaire multi classes) 2DV (voire 3D) doit être envisagé pour une étude relativement plus réaliste des écoulements en régime avec hélices. 
Thème 2 - Dynamique sédimentaire et transports des particules
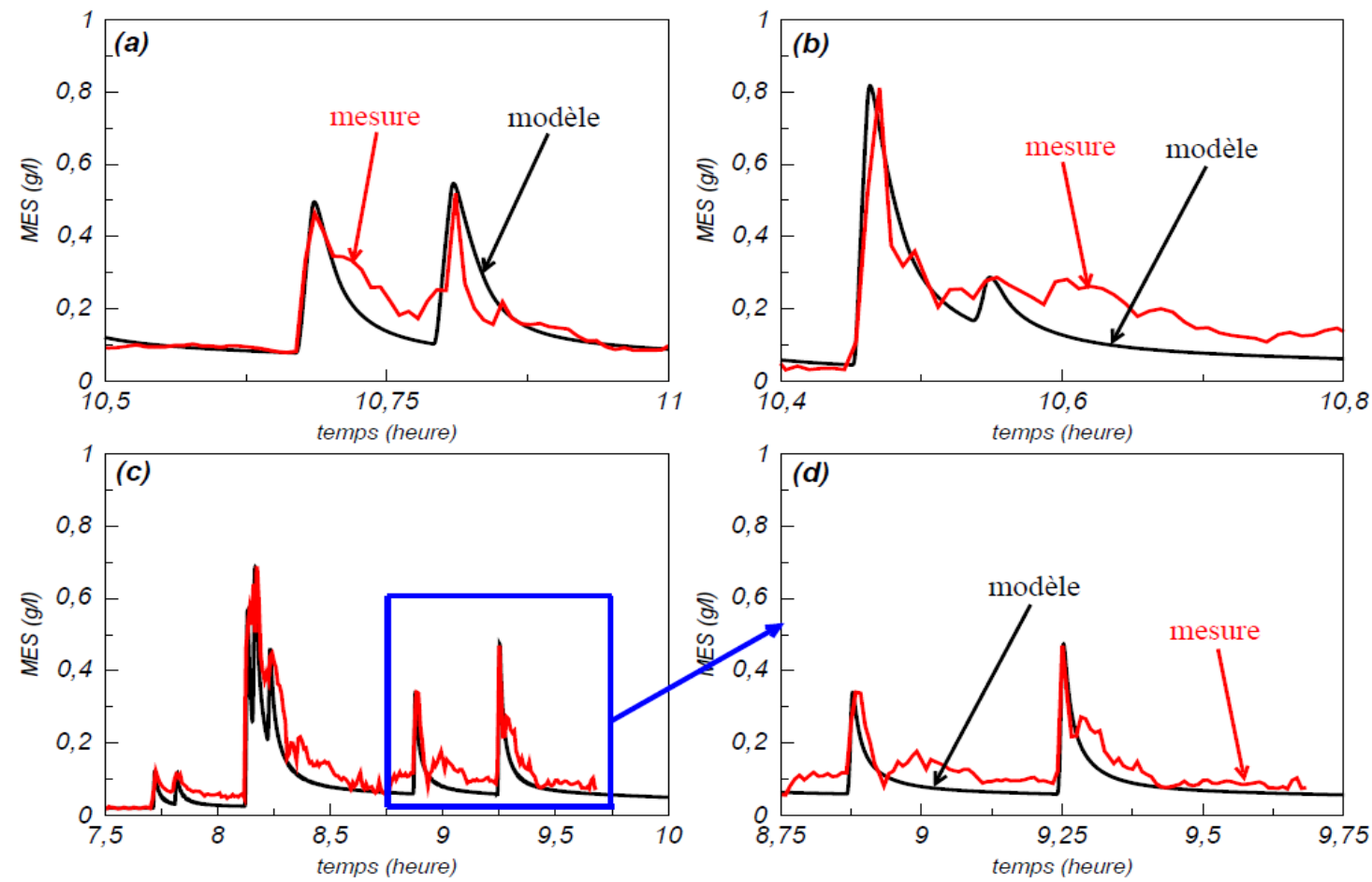

Figure 2. La chronique de la MES issue de la simulation. (a) les pics de la MES induits par les bateaux D6 et D7 de la journée du 08/11/2006, (b) les pics de la MES induits par le bateau D25 de la journée du 09/11/2006, (c) les pics de la MES induits par l'ensemble du trafic fluvial du 10/11/2006 et (d) zoom sur la MES induits par les bateaux D58 et D59 de la journée du 10/11/2006.

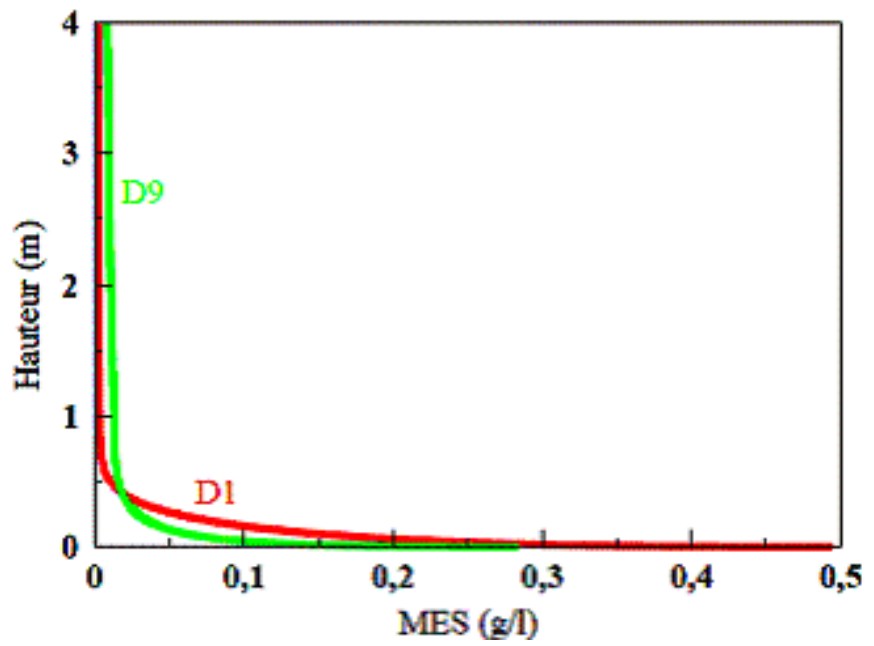

Figure 3. Profils verticaux issus de la simulation de la MES de la journée du 08/11/2006. 


\section{Remerciements}

Les auteurs remercient les Voies Navigables de France (VNF), l'Université du Québec et le Laboratoire Régional de l'Est Parisien (LREP) pour leur soutien financier et technique lors des mesures sur le terrain.

\section{Références bibliographiques}

BLUMBERG A.F., MELLOR G.L. (1987). A description of a three-dimensional coastal ocean circulation model. In: N.S. Heaps (Ed), Three-Dimensional Coastal Ocean Models, Coastal and Estuarine Sciences. 4 A.G.U., Washington (D.C.), pp 1-16. CELIK I., RODI W. (1984). A deposition-entrainment model for suspended sediment transport. Report SFB 210/T/6, University of Karlsruhe (FRG), Karlsruhe, Germany, $55 \mathrm{p}$.

CELIK I., RODI W. (1988). Modeling suspended sediment-transport in nonequilibrium situations. Journal of Hydraulic Engineering, ASCE,114(10), pp 1157-1191. doi:10.1061/(ASCE)0733-9429(1988)114:10(1157)

CELIK I., RODI W. (1991). Suspended sediment-transport capacity for open channel flow. Journal of Hydraulic Engineering, ASCE,117(2), pp 191-204. doi:10.1061/(ASCE)07339429(1991)117:2(191)

PARCHURE T.M, MCANALLY W.H., TEETER A.M. (2001). Desktop method for estimation vessel-induced sediment suspension. Journal of Hydraulic Engineering, ASCE, 127(7), pp 577-587. doi:10.1061/(ASCE)0733-9429(2001)127:7(577)

PHAM VAN BANG D., OUAHSINE A., SERGENT P. HISSEL F. (2007). Impact du passage des bateaux sur le transport des sédiments et l'entretien des voies navigables : mesures et analyses. Rapport (VNF,UTC,CETMEF), 63 p.

PHAM VAN BANG, D., OUAHSINE A., SERGENT P., LONG B., MONTREUIL S., DEBAILLON P., HISSEL F. (2008). Erosion des sédiments et vagues générées par le passage des bateaux: mesures et analyses. $\mathrm{X}^{\mathrm{èmes}}$ Journées Nationales Génie CôtierGénie-Civil, Sophia Antipolis, pp 605-614. doi:10.5150/jngcgc.2008.058-P

VAN RIJN L.C. (1986). Mathematical modeling of suspended sediment in non-uniform flows. Journal of Hydraulic Engineering, ASCE, 12(6), pp 1613-1641.

VERNEY R., DELOFFRE J., BRUN-COTTAN J.C., LAFITE R. (2007). The effect of wave-induced turbulence on intertidal mudflats: impact of boat traffic and wind. Continental Shelf Reseach, 27(5), pp 594-612. doi:10.1016j.csr.2006.10.005 
Thème 2 - Dynamique sédimentaire et transports des particules 